\title{
Outpatient CBT for underweight patients with eating disorders: effectiveness within a National Health Service (NHS) Eating Disorders service
}

Article

Published Version

Jenkins, P. E., Morgan, C. and Houlihan, C. (2019) Outpatient CBT for underweight patients with eating disorders:

effectiveness within a National Health Service (NHS) Eating Disorders service. Behavioural and Cognitive Psychotherapy, 47 (2). pp. 217-229. ISSN 1352-4658 doi: https://doi.org/10.1017/S1352465818000449 Available at https://centaur.reading.ac.uk/77109/

It is advisable to refer to the publisher's version if you intend to cite from the work. See Guidance on citing.

To link to this article DOI: http://dx.doi.org/10.1017/S1352465818000449

Publisher: Cambridge University Press

All outputs in CentAUR are protected by Intellectual Property Rights law, including copyright law. Copyright and IPR is retained by the creators or other copyright holders. Terms and conditions for use of this material are defined in the End User Agreement. 


\section{www.reading.ac.uk/centaur}

\section{CentAUR}

Central Archive at the University of Reading

Reading's research outputs online 


\title{
Outpatient CBT for Underweight Patients with Eating Disorders: Effectiveness Within a National Health Service (NHS) Eating Disorders Service
}

\author{
Paul E. Jenkins \\ Oxford Health NHS Foundation Trust, Cotswold House, Warneford Hospital, Oxford OX3 7JX, UK and \\ School of Psychology and Clinical Language Sciences, University of Reading, Reading RG6 6AL, UK
}

\author{
Ceri Morgan
}

Oxford Health NHS Foundation Trust, Cotswold House, Warneford Hospital, Oxford OX3 7JX, UK

\author{
Catherine Houlihan
}

Oxford Health NHS Foundation Trust, Cotswold House, Warneford Hospital, Oxford OX3 7JX and University of Oxford, The Isis Education Centre, Roosevelt Drive, Warneford Hospital, Headington, Oxford OX3 7JX, UK

\begin{abstract}
Background: Underweight eating disorders (EDs) are notoriously difficult to treat, although a growing evidence base suggests that outpatient cognitive behaviour therapy for EDs (CBT-ED) can be effective for a large proportion of individuals. Aims: To investigate the effectiveness of CBT-ED for underweight EDs in a 'real-world' settings. Method: Sixty-three adults with underweight EDs (anorexia nervosa or atypical anorexia nervosa) began outpatient CBT-ED in a National Health Service setting. Results: Fifty-four per cent completed treatment, for whom significant changes were observed on measures of ED symptoms, psychological distress and psychosocial impairment. There was also a large effect on body weight at end-of-treatment. Conclusions: The results suggest that good outcomes can be achieved by the majority of those who complete treatment, although treatment non-completion remains a significant barrier to recovery. Future studies should focus on improving treatment retention, as evidence suggests that CBT-ED in 'real-world' settings is effective.
\end{abstract}

Keywords: eating disorders, anorexia nervosa, cognitive behaviour therapy, effectiveness

Correspondence to Dr Paul E. Jenkins, School of Psychology and Clinical Language Sciences, University of Reading, Reading RG6 6AL, UK. E-mail: pej106@gmail.com 


\section{Introduction}

In the field of eating disorders (EDs), a number of evidence-based treatments have 'earned their stripes', with those based on cognitive behavioural principles often foremost in the treatment of these illnesses in adults (Steiger, 2017). Cognitive behaviour therapy for EDs, known as CBTED (e.g. NICE, 2017), has been widely investigated and its efficacy supported in randomized controlled trials and meta-analyses (e.g. see Linardon et al., 2017). However, there is often assumed to be a gap between what is provided in research settings (where the majority of evidence for efficacy is collected) and in 'real-world' settings (e.g. see Shafran et al., 2009; Wallace and von Ranson, 2012). In order to address this, a number of recent studies have looked at the effectiveness of evidence-based CBT-ED for adults in outpatient clinical settings (e.g. Byrne et al., 2011; Knott et al., 2015; Rose and Waller, 2017; Serpell et al., 2013; Signorini et al., 2017; Turner et al., 2015; Waller et al., 2014). Such studies are important as they can support findings of well-conducted research trials (e.g. Byrne et al., 2017; Fairburn et al., 2009, 2013, 2015) and demonstrate treatment efficacy in settings where 'there are few exclusion criteria and where adherence to evidence-based protocols is less intensively monitored' (Turner et al., 2015, p. 73).

Of those studies conducted on individual CBT-ED, significant improvements have been reported. Around 50-60\% of patients complete treatment (Byrne et al., 2011; Knott et al., 2015; Turner et al., 2015), with medium to large effect sizes observed on measures of eating pathology and general psychiatric symptoms. Symptom change, commonly assessed with the self-report EDE-Q (Eating Disorder Examination-Questionnaire; Fairburn and Beglin, 2008), suggests that CBT-ED improves ED attitudes as well as behaviours such as binge eating and purging. Similarly, there is growing evidence that effects extend beyond symptom change and are associated with improvements in quality of life (Linardon and Brennan, 2017), although many studies have not included measures of quality of life or psychosocial impairment (e.g. Rose and Waller, 2017).

Studies relating to the treatment of anorexia nervosa (AN) are important given the high treatment costs and the physical and psychological co-morbidities associated with this illness (Agras et al., 2004; Byrne et al., 2017; Halmi et al., 2005). Furthermore, atypical AN (i.e. seen in individuals with clinically significant symptoms for whom one or more of the key symptoms of AN, but not necessarily low weight, is absent; Fairburn and Walsh, 2002) has been understudied (e.g. Santonastaso et al., 2009) and assessing outcome can be complex (Hughes et al., 2017). An additional component of interventions for low weight EDs, such as AN, is the focus on weight restoration as a key goal of treatment, which often necessitates longer duration of treatment (e.g. Byrne et al., 2017). Evaluation of CBTED for AN-type disorders in 'real-world' settings has been limited, with such individuals often only included as part of a larger sample (e.g. Byrne et al., 2011; Rose and Waller, 2017; Signorini et al., 2017; Turner et al., 2015). These shortcomings are also compounded by the fact that sample sizes in treatment studies of AN are generally small, averaging around 20 (Fairburn et al., 2013; see also Linardon et al., 2017). However, two recent trials (Byrne et al., 2017; Fairburn et al., 2013) have reported outcomes for underweight individuals receiving CBT-ED, and generally support findings from non-underweight studies. Thus, although CBT-ED can be applied as a 'transdiagnostic' treatment (Fairburn, 2008), it is important to generalize efficacy of such approaches to low-weight patients in 'real-world' settings. 
The current study aims to describe engagement and outcomes in a sample of consecutively treated adults with underweight EDs. Specifically, it will address four key questions (Fairburn et al., 2013): (1) what proportion of patients with underweight EDs complete CBT-ED in 'realworld' clinical settings?; (2) what is the outcome of patients who complete treatment?; (3) does psychosocial impairment also change following treatment?; and (4) do any baseline variables predict treatment completion? Finally, given recent interest in duration of psychological treatments for EDs (e.g. Bell et al., 2017; Waller et al., 2018), we were also interested in the duration of treatment, and whether the number of treatment sessions was associated with outcome.

\section{Method}

\section{Participants}

The study was conducted within a specialist outpatient Eating Disorders Service within the UK National Health Service (NHS). The service provides assessment and treatment to adults (aged over $17 \frac{1}{2}$ years) ordinarily resident within a local catchment area (total population of around 1.3 million). Patients must first be referred to the service by another healthcare professional, such as a general practitioner, and are then offered treatment at no charge.

Participants were included in the study if they: (a) received an ICD-10 diagnosis of AN or atypical AN (WHO, 2016) following assessment with the service; (b) had a body mass index (BMI) $<18.5$ at start of treatment; (c) had a complete set of self-report measures at start of treatment; (d) subsequently received at least one session of outpatient CBT-ED; (e) were not receiving additional psychological therapy, inpatient or day patient treatment when starting CBT-ED; and (f) started treatment between July 2013 and March 2017. Of note, individuals with a diagnosis of atypical AN were excluded from the study if their BMI was greater than $18.5 \mathrm{~kg} \mathrm{~m}^{-2}$. Approval for conduct of the study was obtained from the local NHS Trust, who noted that further ethical review was not required as this was a retrospective review of routinely collected, de-identified data.

Initial assessment was conducted via clinical interview, lasting approximately 90 minutes. Although similar areas are covered by clinicians, this did not adhere to a standardized interview (e.g. the EDE; Fairburn, 2008). Where ICD-10 (WHO, 2016) diagnosis was unclear, this was agreed upon following team discussion.

The final sample included 62 females and one male with underweight EDs (see Fig. 1). All were asked to complete questionnaires at both pre- and post-treatment. Further demographic information (at start of treatment) is shown in Table 1.

\section{Measures}

Height and weight were measured using a stadiometer and calibrated scales (to the nearest $5 \mathrm{~mm}$ and $0.1 \mathrm{~kg}$, respectively). To assess outcome, the Global score of the EDE-Q (Fairburn and Beglin, 2008) was used to indicate ED pathology and items assessing specific ED behaviours (i.e. objective binge eating, self-induced vomiting, laxatives, and excessive exercise) were also used. This questionnaire also generates a number of subscales (Restraint, Eating Concern, Shape Concern, Weight Concern). Due to significant correlation between the Shape Concern and Weight Concern subscales, a combined subscale (Shape and Weight Concern [SWC]; 


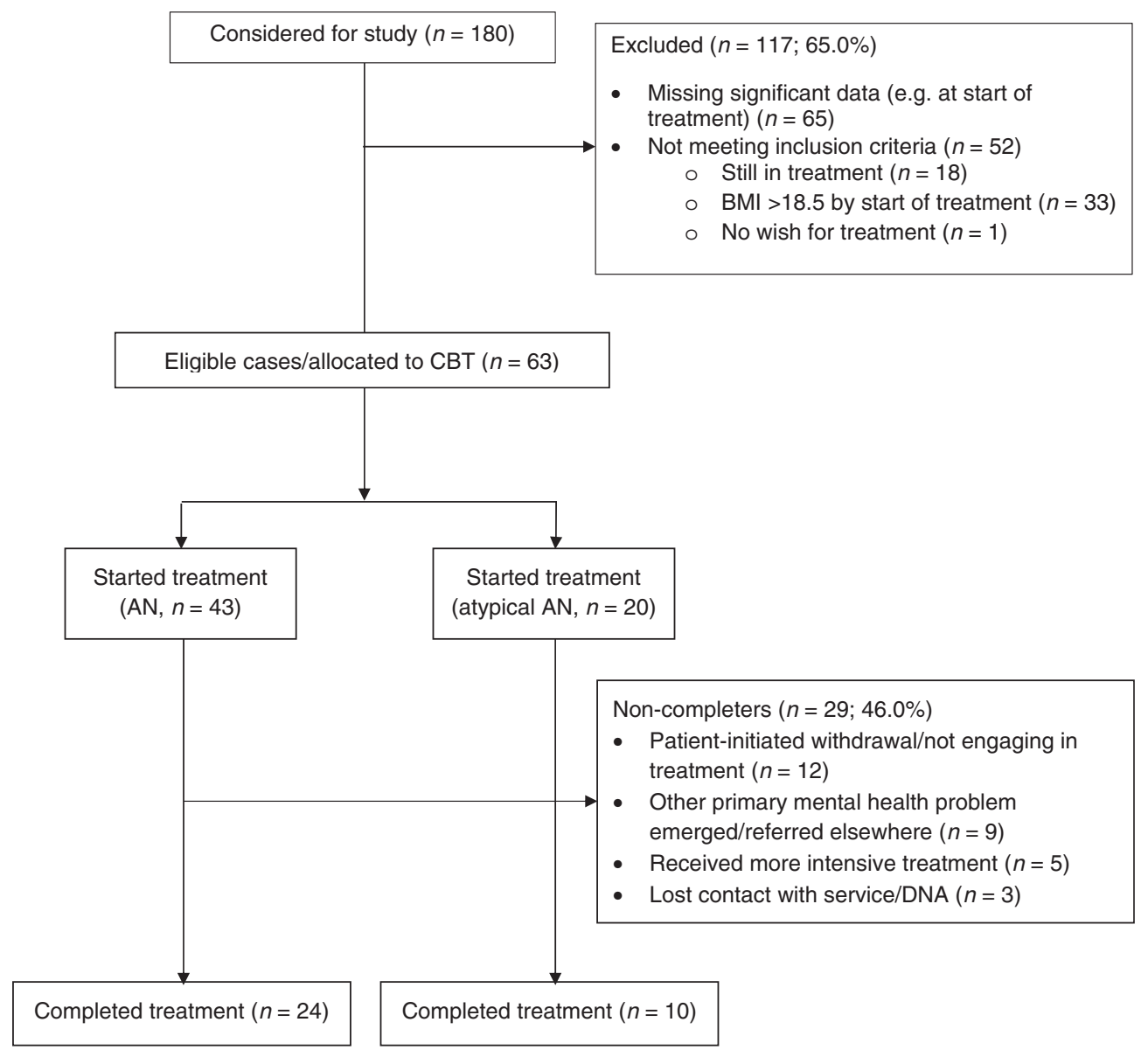

Figure 1. Flow through treatment

$\alpha=0.91$ ) was computed (e.g. Peterson et al., 2007). The Clinical Impairment Assessment Questionnaire (CIA; Bohn and Fairburn, 2008) Total score $(\alpha=0.93)$ was used to assess the degree of psychosocial impairment due to ED pathology and the Clinical Outcomes in Routine Evaluation-Outcome Measure (CORE-OM; Barkham et al., 2001) Total score $(\alpha=0.95)$ provided an index of general psychological distress. All measures have good psychometric properties and have been widely used in ED samples. Additional outcomes included number of sessions attended, number of weeks in treatment, and BMI (calculated as weight [kg] divided by height squared $\left[\mathrm{m}^{2}\right]$ ).

Remission was defined as a BMI $\geq 18.5$, EDE-Q Global score $<2.77$ (one $S D$ above a mean from a community sample of young women; see Fairburn, 2008), and absence of binge eating or dysfunctional weight control behaviours (i.e. self-induced vomiting, laxative use, excessive exercise), which is in line with similar studies (e.g. Byrne et al., 2011; Turner et al., 2015). Partial remission was defined as meeting two of these criteria (e.g. Byrne et al., 2011). 
Table 1. Demographic characteristics of the sample

\begin{tabular}{lccr}
\hline Participant characteristics* & $\begin{array}{c}\text { All } \\
\text { patients }\end{array}$ & $\begin{array}{c}\text { AN } \\
(n=45)\end{array}$ & $\begin{array}{c}\text { Atypical AN } \\
(n=18)\end{array}$ \\
\hline Age, mean $(S D)$, years & $23.63(7.72)$ & $23.32(7.78)$ & $24.39(7.73)$ \\
Ethnic origin $(N=57)$ & & & \\
White-British & $48(84.2 \%)$ & $34(85.0 \%)$ & $14(82.4 \%)$ \\
Other ethnicity & $9(15.8 \%)$ & $6(15.0 \%)$ & $3(17.6 \%)$ \\
Employment status $(N=52)$ & & & \\
Employed & $21(40.4 \%)$ & $14(41.2 \%)$ & $7(38.9 \%)$ \\
Unemployed & $4(7.7 \%)$ & $1(2.9 \%)$ & $8(44.4 \%)$ \\
Full-time student & $26(50.0 \%)$ & $18(52.9 \%)$ & $3(16.7 \%)$ \\
Other & $1(1.9 \%)$ & $1(2.9 \%)$ & - \\
BMI, mean $(S D), \mathrm{kg} \mathrm{m}^{-2}$ & $16.84(1.08)$ & $16.65(1.12)$ & $17.33(0.90)$ \\
Duration of illness, mean $(S D)$, years $(N=44)$ & $6.08(7.38)$ & $5.85(6.93)$ & $6.61(8.64)$ \\
\hline
\end{tabular}

*Numbers differ as data were not available for all participants.

\section{Treatment}

The treatment was a version of CBT-ED based on a leading evidence-based manual (Fairburn, 2008) and resembled that which has been recently recommended by the UK's National Institute for Health and Care Excellence (NICE, 2017). Most elements of Fairburn's (2008) treatment, known as Enhanced CBT (or CBT-E), were followed, including regular collaborative weighing, psychoeducation (including a focus on helping patients decide to change), encouragement of full weight restoration, jointly creating a personalized formulation, regular self-monitoring, addressing core ED features, and sessions becoming less frequent towards the end of treatment. However, the service did not feel able to provide twice-weekly sessions at the outset of treatment but was otherwise consistent in delivering CBT-ED in line with guidance for the psychological treatment of underweight patients (see Fairburn, 2008). ${ }^{1}$ Involvement of significant others (e.g. parent, partner) was considered when it was felt that doing so would facilitate progress (Fairburn, 2008).

Adherence was not closely monitored, although all clinicians were encouraged to deliver treatment as per the manual and were supported by both individual and group supervision. Group supervision took place for one hour per fortnight and focused on key elements of the treatment manual (which was consulted at each session), helping to ensure that treatment procedures were delivered consistently. Individual supervision was provided by one of three senior clinical psychologists, in line with practice recommendations for case management (typically around one hour per month). In total, 10 clinicians delivered treatment and all had attended training courses in CBT-E run by the manual's author.

In line with guidance for CBT-ED (Fairburn, 2008; NICE, 2017), up to 40 sessions were planned. However, this was shortened in the event of rapid change and also if patients no longer wished to continue with treatment or could not routinely commit to making changes (e.g. weight restoration). Although some patients saw another member of the team (e.g. dietitian)

\footnotetext{
${ }^{1}$ Following internal discussion, the Service moved to providing twice-weekly sessions and, anecdotally, this was experienced as positive by both patients and staff.
} 
for a review, none had additional psychological therapy. Those who required more intensive treatment (e.g. inpatient admission) following commencement of CBT-ED are included but classed as non-completers.

As in previous work, 'non-completion' was defined as 'the patient ending treatment before the agreed termination point (defined by patient and clinician)' (Waller et al., 2014, p. 14); all other patients were deemed to have completed a course of treatment (see Fig. 1). The majority of individuals completed treatment earlier than the planned 40 sessions; typically, patients had completed the relevant steps of treatment and experienced sufficient improvement in symptoms. Six individuals $(9.5 \%)$ received more than 40 sessions, agreed in supervision, all of whom were treatment completers.

\section{Statistical analyses}

To obtain outcomes based on intent-to-treat (ITT) analysis, multiple imputation (MI) with chained equations was conducted for missing data on BMI, Global EDE-Q, SWC, CIA Total, and CORE-OM Total. Questionnaire responses were imputed at scale level given high internal consistency and construction from many items (Graham, 2009). Square-root transformation reduced the degree of non-normality, so transformed variables formed the basis for analysis (back-transformed to aid interpretation of results). Transformation did not correct non-normality in distribution of ED behaviours (e.g. binge eating), so MI was conducted on untransformed data given the preference for MI to other missing data methods (Graham, 2009).

The imputation model contained pre-treatment age and BMI as predictors and was based on linear regression for continuous variables. Twenty datasets for each outcome variable were generated. A similar procedure was carried out for ED behaviours, with the exception that imputations were estimated from age, BMI and separate ED behaviours. Cohen's $d$ is reported as a measure of effect size, obtained from paired samples $t$-tests for pre-post analysis.

In order to identify predictors of treatment completion, baseline variables were compared with independent samples $t$-tests, followed by logistic regression analysis (forward entry) with significant variables. The model was run with backward entry, but this did not affect the results.

To investigate the effect of treatment duration, partial correlations (controlling for EDE-Q and BMI at pre-treatment) were used to assess the relationship between number of sessions and BMI change (post-treatment BMI minus pre-treatment BMI) and EDE-Q at post-treatment (transformed data) (e.g. see Bell et al., 2017).

\section{Results}

\section{Intent-to-treat analysis}

Looking at the sample as a whole, there was a significant main effect of treatment on all variables (see Table 2). At the end of treatment, nine individuals (14.3\%) met criteria for full remission with a further $22(34.9 \%)$ partial remission; 22 of these $(71.0 \%)$ were treatment completers. Specific numbers regarding each remission criterion are provided in Table 2.

Looking at diagnostic differences, Table 3 presents further information for AN and atypical AN groups. No significant differences were found between the two groups. 
Table 2. Changes in symptomatology over the course of treatment.

\begin{tabular}{|c|c|c|c|c|c|c|c|c|c|c|}
\hline \multirow[b]{3}{*}{ Measure } & \multicolumn{5}{|c|}{ Treatment completers $(n=34)$} & \multicolumn{5}{|c|}{ All participants $(n=63)$} \\
\hline & \multicolumn{3}{|c|}{ Mean (standard error) } & \multirow[b]{2}{*}{ Cohen's $d$} & \multirow[b]{2}{*}{$p$} & \multicolumn{3}{|c|}{ Mean (standard error) } & \multirow[b]{2}{*}{ Cohen's $d$} & \multirow[b]{2}{*}{$p$} \\
\hline & Pre & Post & $t$ & & & Pre & Post & $t$ & & \\
\hline BMI & $17.04(0.18)$ & $19.14(0.25)$ & -6.97 & 1.72 & $<.001$ & $16.86(0.14)$ & $18.24(0.23)$ & -8.14 & 1.46 & $<.001$ \\
\hline Global EDE-Q & $3.90(0.19)$ & $2.01(0.25)$ & 5.95 & 1.46 & $<.001$ & $3.71(0.16)$ & $2.07(0.21)$ & 6.52 & 1.17 & $<.001$ \\
\hline SWC & $4.12(0.22)$ & $2.45(0.29)$ & 5.15 & 1.27 & $<.001$ & $4.01(0.18)$ & $2.48(0.22)$ & 5.75 & 1.03 & $<.001$ \\
\hline CORE Total & $18.34(1.14)$ & $11.68(1.30)$ & 4.26 & 1.05 & $<.001$ & $18.79(0.86)$ & $12.50(1.16)$ & 4.53 & 0.81 & $<.001$ \\
\hline CIA Total & $31.17(1.72)$ & $14.82(2.14)$ & 5.66 & 1.39 & $<.001$ & $31.94(1.315)$ & $16.01(1.83)$ & 6.34 & 1.16 & $<.001$ \\
\hline \multicolumn{11}{|l|}{ Remission outcome } \\
\hline Global EDE-Q <2.77 & $6(17.6 \%)$ & $25(73.5 \%)$ & - & - & - & $16(25.4 \%)$ & $46(73.0 \%)$ & - & - & - \\
\hline $\mathrm{BMI}>18.5$ & - & $23(67.6 \%)$ & - & - & - & - & $30(47.6 \%)$ & - & - & - \\
\hline $\begin{array}{l}\text { Absence of } \\
\text { behavioural } \\
\text { symptoms }\end{array}$ & $5(14.7 \%)$ & $12(35.3 \%)$ & - & - & - & $13(20.6 \%)$ & $18(28.6 \%)$ & - & - & - \\
\hline Full remission & - & $7(20.6 \%)$ & - & - & - & - & $9(14.3 \%)$ & - & - & - \\
\hline Partial remission & - & $15(44.1 \%)$ & - & - & - & $5(7.9 \%)$ & $22(34.9 \%)$ & - & - & - \\
\hline
\end{tabular}

*Sum of: objective binge eating episodes, self-induced vomiting, laxative use, excessive exercise; $n=27$ (completers) and $n=54$ (ITT). $¥ n=33$ (completers) and $n=62$ (ITT) as one individual did not complete this measure at start-of-treatment. 
Table 3. Differences in outcome in anorexia nervosa (AN) and atypical AN (ITT)

\begin{tabular}{lccrc}
\hline & AN $(n=45)$ & Atypical AN $(n=18)$ & Test statistic & $p$-value \\
\hline Treatment non-completers, $N(\%)$ & $20(44.4 \%)$ & $9(50.0 \%)$ & $\chi^{2}=0.160$ & 0.689 \\
EDE-Q Global change*, mean $(S E)$ & $1.76(0.27)$ & $1.48(0.51)$ & $t=-0.525$ & 0.600 \\
BMI change*, mean $(S E)$ & $1.31(0.27)$ & $1.24(0.28)$ & $t=0.161$ & 0.872 \\
Remission outcome & & & & \\
Global EDE-Q $<2.77, N(\%)$ & $33(73.3 \%)$ & $13(72.2 \%)$ & $\chi^{2}=0.008$ & 0.928 \\
BMI $>18.5, N(\%)$ & $20(44.4 \%)$ & $10(55.6 \%)$ & $\chi^{2}=0.636$ & 0.425 \\
\hline
\end{tabular}

${ }^{*}$ Change in score from pre-treatment to post-treatment.

\section{Treatment completion}

Twenty-nine individuals (46.0\%) did not complete CBT-ED. On average, patients received 23.1 sessions of CBT-ED (range $=3$ to 61 ) over 30.4 weeks ( 2 to 75 ). Completers attended more sessions that non-completers $(30.6$ vs $14.4, t(61)=6.591, p<.001)$.

\section{Treatment duration}

Of note, $76 \%$ of those deemed to have completed CBT-ED did not require 40 sessions. Partial correlations (controlling for pre-treatment EDE-Q Global and BMI) were significant between number of treatment sessions and BMI change $(r=.474, p=.003)$ but not EDE-Q Global at post-treatment $(r=-.110, p=.522)$.

\section{Outcomes of treatment completers}

For those who completed a course of CBT-ED, there was a considerable response on a number of symptom measures, with large effect sizes (see Table 2). Mean BMI gain was $2.11 \mathrm{~kg} \mathrm{~m}^{-2}$ $(\mathrm{SE}=0.27)$ and around two-thirds $(23 / 34 ; 67.6 \%)$ achieved a BMI $\geq 18.5$. This change was significantly greater than the $+0.50 \mathrm{~kg} \mathrm{~m}^{-2}(S E=0.21)$ for non-completers $(t(4044)=4.627$, $p<.001)$. There was also significant improvement on a measure of psychological distress, and positive changes in ED behaviours. Seven individuals (20.6\%) met criteria for full remission with a further $15(44.1 \%)$ for partial remission.

\section{Effects on psychosocial impairment}

Mean CIA scores had decreased significantly at end-of-treatment, with a large effect size. The mean score for completers at end-of-treatment was just below the cut-off for case status of 16 (Bohn et al., 2008), and 20 (58.8\%) scored below this. For the intent-to-treat sample, the mean was slightly higher and $37(58.7 \%)$ scored below the cut-off.

\section{Predictors of treatment completion}

Comparison at baseline (start-of-treatment) revealed no differences between those who completed treatment and those who did not on BMI, SWC, EDE-Q Global, CORE Total or CIA Total scores. Presence and frequency (two separate variables) of binge eating and 
purging did differ, with completers more likely to experience binge eating and purging $\left(\chi^{2}(1)=6.833, p=.009\right)$ and more episodes of binge eating and purging (23.7 vs 4.93, $t(52)=2.927, p=.006)$. This variable remained significant in regression analyses whether considered as a binary (present/not) or continuous variable. Median-centring (see Kraemer and Blasey, 2004) produced identical results.

\section{Discussion}

Underweight EDs represent some of the most difficult psychiatric illnesses to treat, although emerging evidence suggests that many patients are able to make significant gains in outpatient treatment (e.g. Fairburn et al., 2013). Application to 'real-world' samples is formative, in part as existing case series have largely included individuals with AN only as part of larger analyses. However, this study complements well-conducted trials (e.g. Byrne et al., 2017; Fairburn et al., 2013) in reporting outcomes for adults with low-weight EDs following outpatient psychological treatment in a 'real-world' setting.

The proportion of individuals who completed treatment in the current study (54\%) is comparable to other 'real-world' studies (e.g. Signorini et al., 2017: 50\%; Turner et al., 2015: $55 \%$ ) and is slightly smaller than RCTs for underweight patients (e.g. Byrne et al., 2017: 66.7\%; Fairburn et al., 2013: 64\%). As this sample wholly consisted of individuals who were underweight, results suggest that completion rates for CBT-ED may not differ greatly across diagnoses, although larger studies will be needed to test this assumption. Of note, treatment completers received an average of around 31 sessions of CBT-ED, far less than typically recommended (e.g. Fairburn, 2008). A complete explanation for this is unclear, given that adherence was not assessed, although it has been suggested that it might be possible to develop briefer versions of CBT-ED, even for those who are underweight, or for certain patients to move through the stages of treatment more rapidly than others (Waller et al., 2018). For example, Byrne et al. (2017) amended length of treatment for AN to reflect the magnitude of weight restoration required, although no such modification was explicitly applied in the current study. Partial support for this endorsement comes from the fact that duration of treatment was related only to change in BMI, and not ED pathology, suggesting that those who remained in treatment longer restored more weight (cf. Bell et al., 2017; Rose and Waller, 2017). However, as outcomes were not recorded other than at pre- and post-treatment, more detailed analysis (e.g. of session-by-session change) would be beneficial to determine the optimal number of sessions required for significant change within CBT-ED.

Duration of illness in the current sample was longer than that of both Fairburn and colleagues (2013) and Byrne et al. (2017), although other pre-treatment characteristics (e.g. gender ratio, mean BMI, degree of psychosocial impairment) were comparable. Given such similarities, existing data repeatedly points to the finding that attrition in 'real-world' samples exceeds that in research settings, and is related to poorer outcome overall (e.g. Byrne et al., 2011).

In terms of weight restoration - a key goal for remission from underweight EDs CBT-ED completers restored just over two BMI points from start to end of treatment. In terms of remission rates, a BMI of at least $18.5 \mathrm{~kg} \mathrm{~m}^{-2}$ was achieved by over $60 \%$ of treatment completers in Fairburn et al. (2013) and over 50\% of the total sample in Byrne et al. (2017). Minimal ED pathology was achieved by $80 \%$ of those who completed treatment in Fairburn et al. (2013). In the current study, over two-thirds of completers achieved weight restoration 
(just under half of the ITT sample) and nearly three-quarters reported minimal ED pathology. Overall, results are encouraging given the low body weight (BMI range at start of treatment = 14.8 to 18.5 ) and lack of strict treatment monitoring, although more needs to be done to improve outcome for all patients given the smaller treatment effects in ITT analyses. Close adherence to evidence-based CBT-ED is a possible explanation for the positive results (Linardon et al., 2017), although such interpretations should be cautious as no measure of adherence was included.

Other secondary outcomes were also promising, with levels of psychological distress significantly improved following treatment, in addition to reported impairment as a result of ED pathology (see also Byrne et al., 2017). Therefore, findings support existing data that those who complete treatment report better outcomes than those who do not (e.g. see Fairburn et al., 2015). However, given that positive results were also seen in the ITT condition (with around half achieving at least partial remission), problems around impaired access to psychological treatments (see Kazdin et al., 2017) and treatment completion also demand resolution.

Only one variable emerged as a significant predictor of outcome - presence of binge eating and purging was positively associated with treatment completion. However, this was highly unexpected given results of both non-randomized studies (e.g. Fairburn et al., 2013) and reviews (e.g. Vall and Wade, 2015) which suggest that higher levels of these behaviours are associated with worse outcome. Although this finding could be due to limitations of the study, such as a small number of non-completers who engaged in binge eating and purging at the start of treatment $(n=4)$ and lack of randomization, other explanations are possible. It could be, for example, that individuals were motivated to address this behaviour as part of CBT-ED (e.g. Vitousek et al., 1998) and therefore remained in treatment. It is also possible that the early focus on reducing binge eating and purging was associated with positive outcomes (e.g. see Brown et al., 2013). Other studies (e.g. Turner et al., 2015) have, however, failed to find that the presence of binge eating and purging predicts outcome and therefore more study is warranted, perhaps considering the timing of any change in these symptoms.

Limitations of the current study include the small number of male cases and use of selfreport data in assessing both symptomatology and impairment. Although the sample size was favourable compared with other studies of AN, this was too small to conduct detailed statistical analyses, such as moderator and mediator effects and certain subgroup analyses (e.g. presence of binge eating and purging). Although we considered diagnostic differences, it was not possible to look at outcomes of different groups subtyped by behavioural symptoms. Use of ICD-10 criteria (WHO, 2016), which do not include presence of purging as an essential criterion for AN diagnosis, also limited this possibility. One further weakness of the study is also perhaps its major strength. The CBT-ED conducted, whilst closely aligned to a manual (Fairburn, 2008), was not uniform; a number of therapists delivered the treatment and the number of sessions received by completers varied from 17 to 61 . However, this also speaks to existing evidence that CBT-ED can be delivered effectively in 'real-world' settings when core strategies are followed (Rose and Waller, 2017; Turner et al., 2015; Waller et al., 2018).

Overall the results presented here suggest that individuals with AN and atypical AN who are underweight can be treated effectively with CBT-ED in a 'real-world' setting. Outcomes are similar to RCTs and, taken together, suggest that evidence-based treatments can be readily applied with individuals with EDs in 'real-world' settings. As Turner et al. (2015) conclude, the focus should now be on enhancing retention in evidence-based treatments, given that those who adhere to treatment (i.e. completers) generally report good clinical outcomes. 
Acknowledgements: The authors would like to acknowledge the therapists and other staff for their support with this work.

Ethical statements: The authors assert that they have abided by the Ethical Principles of Psychologists and Code of Conduct as set out by the APA. Approval for conduct of the study was obtained from the local NHS Trust, who noted that further ethical review was not required as this was a retrospective review of routinely collected, de-identified data.

Conflicts of interest: The authors declare no conflicts of interest with respect to this publication.

Financial support: This research received no specific grant from any funding agency, commercial or not-for-profit sectors.

\section{References}

Agras, W. S., Brandt, H. A., Bulik, C. M., Dolan-Sewell, R., Fairburn, C. G., Halmi, K. A. et al. (2004). Report of the National Institutes of Health workshop on overcoming barriers to treatment research in anorexia nervosa. International Journal of Eating Disorders, 35, 509-521. doi: 10.1002/eat.10261

Barkham, M., Margison, F., Leach, C., Lucock, M., Mellor-Clark, J., Evans, C. et al. (2001). Service profiling and outcomes benchmarking using the CORE-OM: toward practice-based evidence in the psychological therapies. Journal of Consulting and Clinical Psychology, 69, 184-196. doi: 10.1037/0022-006X.69.2.184

Bell, C., Waller, G., Shafran, R. and Delgadillo, J. (2017). Is there an optimal length of psychological treatment for eating disorder pathology? International Journal of Eating Disorders, 50, 687-692. doi: 10.1002/eat.22660

Bohn, K. and Fairburn, C. G. (2008). Clinical Impairment Assessment Questionnaire (CIA 3.0). In C. G. Fairburn (ed), Cognitive Behaviour Therapy and Eating Disorders (pp. 315-317). New York: Guilford Press.

Brown, A., Mountford, V. and Waller, G. (2013). Therapeutic alliance and weight gain during cognitive behavioural therapy for anorexia nervosa. Behaviour Research and Therapy, 51, 216-220. doi: 10.1016/j.brat.2013.01.008

Byrne, S. M., Fursland, A., Allen, K. L. and Watson, H. (2011). The effectiveness of enhanced cognitive behavioural therapy for eating disorders: an open trial. Behaviour Research and Therapy, 49, 219-226. doi: 10.1016/j.brat.2011.01.006

Byrne, S., Wade, T., Hay, P., Touyz, S., Fairburn, C. G., Treasure, J. et al. (2017). A randomised controlled trial of three psychological treatments for anorexia nervosa. Psychological Medicine, 47, 2823-2833. doi: https://doi.org/10.1017/S0033291717001349

Fairburn, C. G. (2008). Cognitive Behavior Therapy and Eating Disorders. New York: Guilford Press.

Fairburn, C. G. and Beglin, S. J. (2008). Eating Disorder Examination Questionnaire (EDE-Q 6.0). In C. G. Fairburn (ed), Cognitive Behaviour Therapy and Eating Disorders (pp. 309-313). New York: Guilford Press.

Fairburn, C. G., Cooper, Z., Doll, H. A., O'Connor, M. E., Bohn, K., Hawker, D. M. et al. (2009). Transdiagnostic Cognitive-Behavioral Therapy for patients with eating disorders: a two-site trial with 60-week follow-up. American Journal of Psychiatry, 166, 311-319. doi: 10.1176/appi.ajp.2008.08040608

Fairburn, C. G., Cooper, Z., Doll, H. A., O'Connor, M. E., Palmer, R. L. and Dalle Grave, R. (2013). Enhanced cognitive behaviour therapy for adults with anorexia nervosa: a UK-Italy study. Behaviour Research and Therapy, 51, R2-R8. doi: 10.1016/j.brat.2012.09.010 
Fairburn, C. G., Bailey-Straebler, S., Basden, S., Doll, H. A., Jones, R., Murphy, R. et al. (2015). A transdiagnostic comparison of enhanced cognitive behaviour therapy (CBT-E) and interpersonal psychotherapy in the treatment of eating disorders. Behaviour Research and Therapy, 70, 64-71. doi: 10.1016/j.brat.2015.04.010

Fairburn, C. G. and Walsh, B. T. (2002). Atypical eating disorders (Eating Disorder Not Otherwise Specified). In C. G. Fairburn and K. D. Brownell (eds), Eating Disorders and Obesity: A Comprehensive Handbook (2nd edn, pp. 171-177). New York: Guilford Press.

Graham, J. W. (2009). Missing data analysis: making it work in the real world. Annual Review of Psychology, 60, 549-576. doi: 10.1146/annurev.psych.58.110405.085530

Halmi, K. A., Agras, W. S., Crow, S., Mitchell, J., Wilson, G. T., Bryson, S. and Kraemer, H. C. (2005). Predictors of treatment acceptance and completion in anorexia nervosa: implications for future study designs. Archives of General Psychiatry, 62, 776-781. doi: 10.1001/archpsyc.62.7.776

Hughes, E. K., Le Grange, D., Court, A. and Sawyer, S. M. (2017). A case series of family-based treatment for adolescents with atypical anorexia nervosa. International Journal of Eating Disorders, 50, 424-432. doi: 10.1002/eat.22662

Kazdin, A. E., Fitzsimmons-Craft, E. E. and Wilfley, D. E. (2017). Addressing critical gaps in the treatment of eating disorders. International Journal of Eating Disorders, 50, 170-189. doi: 10.1002/eat. 22670

Knott, S., Woodward, D., Hoefkens, A. and Limbert, C. (2015). Cognitive behaviour therapy for bulimia nervosa and eating disorders not otherwise specified: translation from randomized controlled trial to a clinical setting. Behavioural and Cognitive Psychotherapy, 43, 641-654. doi: $10.1017 /$ S1352465814000393

Kraemer, H. C. and Blasey, C. M. (2004). Centring in regression analyses: a strategy to prevent errors in statistical inference. International Journal of Methods in Psychiatric Research, 13, 141-151. doi: 10.1002/mpr.170

Linardon, J. and Brennan, L. (2017). The effects of cognitive-behavioral therapy for eating disorders on quality of life: a meta-analysis. International Journal of Eating Disorders, 50, 715-730. doi: 10.1002/eat.22719

Linardon, J., Wade, T. D., De La Piedad Garcia, X. and Brennan, L. (2017). The efficacy of cognitivebehavioral therapy for eating disorders: a systematic review and meta-analysis. Journal of Consulting and Clinical Psychology, 85, 1080-1094. doi: 10.1037/ccp0000245

National Institute for Health and Care Excellence (NICE) (2017). Eating disorders: recognition and treatment. NICE guideline (NG69). London, UK: NICE.

Peterson, C. B., Crosby, R. D., Wonderlich, S. A., Joiner, T., Crow, S. J., Mitchell, J. E. et al. (2007). Psychometric properties of the Eating Disorder Examination-Questionnaire: factor structure and internal consistency. International Journal of Eating Disorders, 40, 386-389. doi: 10.1002/eat.20373

Rose, C. and Waller, G. (2017). Cognitive-behavioral therapy for eating disorders in primary care settings: does it work, and does a greater dose make it more effective? International Journal of Eating Disorders, 50, 1350-1355. doi: 10.1002/eat.22778

Santonastaso, P., Bosello, R., Schiavone, P., Tenconi, E., Degortes, D. and Favaro, A. (2009). Typical and atypical restrictive anorexia nervosa: weight history, body image, psychiatric symptoms, and response to outpatient treatment. International Journal of Eating Disorders, 42, 464-470. doi: 10.1002/eat.20706

Serpell, L., Stobie, B., Fairburn, C. G. and van Schaick, R. (2013). Empirically-supported and nonempirically supported therapies for bulimia nervosa: retrospective patient ratings. Journal of Eating Disorders, 1, 41. doi: 10.1186/2050-2974-1-41

Shafran, R., Clark, D. M., Fairburn, C. G., Arntz, A., Barlow, D. H., Ehlers, A. et al. (2009). Mind the gap: improving the dissemination of CBT. Behaviour Research and Therapy, 47, 902-909. doi: 10.1016/j.brat.2009.07.003 
Signorini, R., Sheffield, J., Rhodes, N., Fleming, C. and Ward, W. (2017). The effectiveness of Enhanced Cognitive Behavioural Therapy (CBT-E): a naturalistic study within an out-patient eating disorder service. Behavioural and Cognitive Psychotherapy, 46, 21-34. doi: $10.1017 / \mathrm{S} 1352465817000352$

Steiger, H. (2017). Evidence-informed practices in the real-world treatment of people with eating disorders. Eating Disorders, 25, 173-181. doi: 10.1080/10640266.2016.1269558

Turner, H., Marshall, E., Stopa, L. and Waller, G. (2015). Cognitive-behavioural therapy for outpatients with eating disorders: effectiveness for a transdiagnostic group in a routine clinical setting. Behaviour Research and Therapy, 68, 70-75. doi: 10.1016/j.brat.2015.03.001

Vall, E. and Wade, T. D. (2015). Predictors of treatment outcome in individuals with eating disorders: a systematic review and meta-analysis. International Journal of Eating Disorders, 48, 946-971. doi: 10.1002/eat.22411

Vitousek, K., Watson, S. and Wilson, G. T. (1998). Enhancing motivation for change in treatment-resistant eating disorders. Clinical Psychology Review, 18, 391-420. doi: 10.1016/S02727358(98)00012-9

Wallace, L. M. and von Ranson, K. M. (2012). Perceptions and use of empirically-supported psychotherapies among eating disorder professionals. Behaviour Research and Therapy, 50, 215222. doi: $10.1016 /$ j.brat.2011.12.006

Waller, G., Gray, E., Hinrichsen, H., Mountford, V., Lawson, R., and Patient, E. (2014). Cognitivebehavioral therapy for bulimia nervosa and atypical bulimic nervosa: effectiveness in clinical settings. International Journal of Eating Disorders, 47, 13-17. doi: 10.1002/eat.22181

Waller, G., Tatham, M., Turner, H., Mountford, V. A., Bennetts, A., Bramwell, K. et al. (2018). A 10-session cognitive-behavioral therapy (CBT-T) for eating disorders: outcomes from a case series of non-underweight adult patients. International Journal of Eating Disorders, 51, 262-269. doi: 10.1002/eat. 22837

World Health Organization (WHO) (2016). ICD-10 version: 2016. Available at: http://apps.who.int/ classifications/icd10/browse/2016/en\# 